\title{
AN ANALYSIS OF FRETTING FATIGUE CRACKS DURING LOADING PHASE
}

\author{
D. A. Hullst and Maria Comninou \\ Dept. Mechanical Engineering and Applied Mechanics. University of Michigan. Ann Arbor.
} MI 4\$IO9. U.S.A.

\begin{abstract}
An elastic half-plane, subjected to loading by uniform tractions over a given length of its surface, is considered. The tractions consist of pressure, constant in time, and a shear load, varying sinusoidally in lime. The plane also contains a surface-breaking crack. normal to the free surface and located at the cdge of the pressurized region. This geometry approximates the classical fretting problem with a resulting fatigue crack. The faces of the crack are allowed to transmil Coulomb friction.

In this study, the first quarter cycle of loading (shear tractions monotonically increased from zero) is considered. Stress intensity factors are computed for various crack lengths. friction coefficients, and ratios of applied loads.
\end{abstract}

\section{INTRODUCTION}

Fretting occurs when two surfaces are clamped together, but transmit also a transverse shear, typically varying harmonically in time, so that there are some regions of microslip. This microslip, often exacerbated by the ingress of corrosive fluids. causes rapid deterioration of the surfaces, which then provide good sites for the nucleation of fatigue cracks[1]. One of the components is often in the form of a flat "foot" with square corners, so that there is a concentration of the bulk stress field, and this therefore provides a favorable site for crack initiation. Experience shows that a fatigue crack almost invariably grows downwards, approximately vertically, from this point [1]. and may lead to catastrophic failure.

There are essentially two aspects of the problem which require investigation: (1) The traction distribution history between the contacting bodies must be computed, including any zones of slip and locking. This has been examined by Wright and O'Connor [2, 3] for flawless bodies; (2) Given the nominal stress field indicated by the first phase of the solution, it is required to find the stress history as modified by a preexisting crack which is capable of transmitting frictional forces across its faces. The stress intensity factors are particularly of interest. An approximate analysis has been carried out by Edwards[4] for applied loads causing the crack to remain completely open.

The two parts of the solution are actually coupled since the presence of the crack affects the compliance of one of the contacting bodies. An analysis of the fully coupled problem has been presented in [5] using a finite element formulation. The uncoupled problem with a Hertzian contact distribution and surface friction was considered in [6], using integral equations. Neither work considered the effect of friction between the crack faces. The present study considers the latter effect and pays particular attention to the effect of crack length, interfacial friction, and applied shear. To keep the analysis tractable, however, the applied loading is idealized.

The geometry of the problem is shown in Fig. la. A normal load of constant magnitude is applied first, and a shear load increasing from zero to a maximum value, Fig. 1b, is applied subsequently. The remaining part of the loading cycle requires an incremental formulation and is considered in a subsequent paper.

\section{FORMULATION}

In the absence of the crack, the stresses due to uniform pressure of magnitude $p_{0}$. and shear tractions of magnitude $\lambda p_{s}$ (Fig. la), may be obtained by direct integration 
(a).

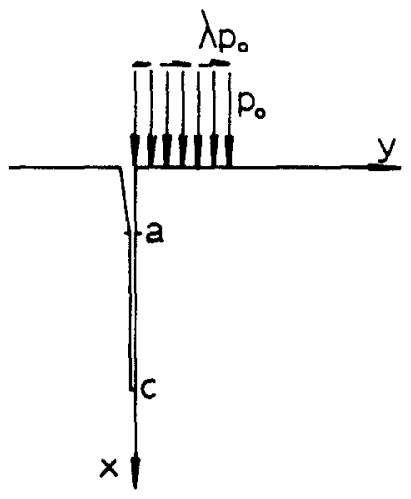

(b).

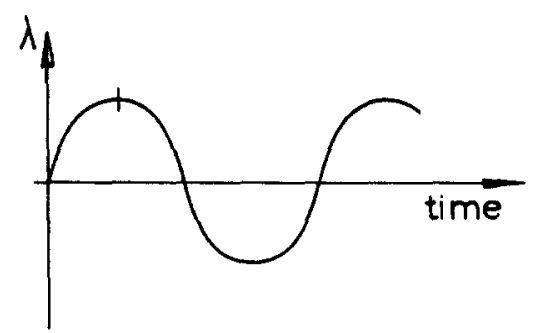

Fig. 1. (a) Crack and Loading geometry; (b) Variation of $\lambda$ with time.

of the Flamant solution[7]. The results on $y=0$ are

$$
\begin{aligned}
& \sigma_{x y} / p_{\prime \prime}=g_{1}+\lambda g_{2} \\
& \sigma_{x y} / p_{1}=g_{2}+\lambda g_{3},
\end{aligned}
$$

where

$$
\begin{aligned}
& g_{1}(x)=\frac{1}{\pi\left(1+x^{2}\right)}>0 \\
& g_{2}(x)=\frac{1}{\pi}\left[\frac{1}{1+x^{2}}-\tan ^{-1}\left(\frac{1}{x}\right)\right]<0 \\
& g_{3}(x)=-\frac{1}{\pi}\left[\frac{1}{1+x^{2}}+\ln \left(\frac{x^{2}}{1+x^{2}}\right)\right]>0 .
\end{aligned}
$$

It is noted that the coordinate $x$ has been normalized with respect to the load length $L$, and that the algebraic signs of the functions are indicated for future reference.

Once the load is applied, it is found that zones of slip and separation develop between the crack faces during the first half of the loading cycle (Fig. 1b). To accommodate these zones, we distribute climb dislocations $B_{y}$ over the separation zone $S_{1}$ and glide dislocations $B_{x}$ over the separation and slip regions $S_{2}$. Using the results of [8] and [9], the total normal tractions on $y=0$ are

$$
\begin{aligned}
& N(x)=\sigma_{y y}+\frac{2 \mu}{\pi(\kappa+1)} \int_{S_{1}} B_{y}(\xi) K(x, \xi) \mathrm{d} \xi \\
& S(x)=\sigma_{x y}+\frac{2 \mu}{\pi(\kappa+1)} \int_{S_{2}} B_{x}(\xi) K(x, \xi) \mathrm{d} \xi,
\end{aligned}
$$


where

$$
K(x, \xi)=\frac{1}{x-\xi}-\frac{1}{x+\xi}-\frac{2 \xi}{(x+\xi)^{2}}+\frac{4 \xi^{2}}{(x+\xi)^{2}}
$$

$\mu$ is the shear modulus and $k=3-4 \nu$ for plane strain, where $\nu$ is Poisson's ratio.

The starting condition for the present problem is that a uniform pressure $p_{v}$, has been applied on the half-plane containing the crack, which is the problem solved in [8]. Here we assume that the coefficient of friction $f$ is sufficiently low so that the crack is initially in a state of forward (positive) slip along its entire length. From [8], this means that $f$ must be less than $f_{\text {max }}$ shown by line A, Fig. 2, which is not, in practice, a restriction. At higher values of $f$ stick between the crack faces starts at the surface. Under conditions of forward slip, the shear traction transmitted by the crack is given by

$$
S(x)=-f N(x)
$$

and the shear stress intensity factor at the crack tip, again from [8], is given by curves $D$, Fig. 2. The shear tractions are now gradually increased from zero, causing separation to start at the surface, and adjacent forward slip or backslip to occur along (part of) the closed crack extent. Since there is no coupling between $B_{x}$ and $B_{y}$ in eqns (6) and (7), we first determine the extent of separation $a$ as a function of $\lambda$, and the resulting

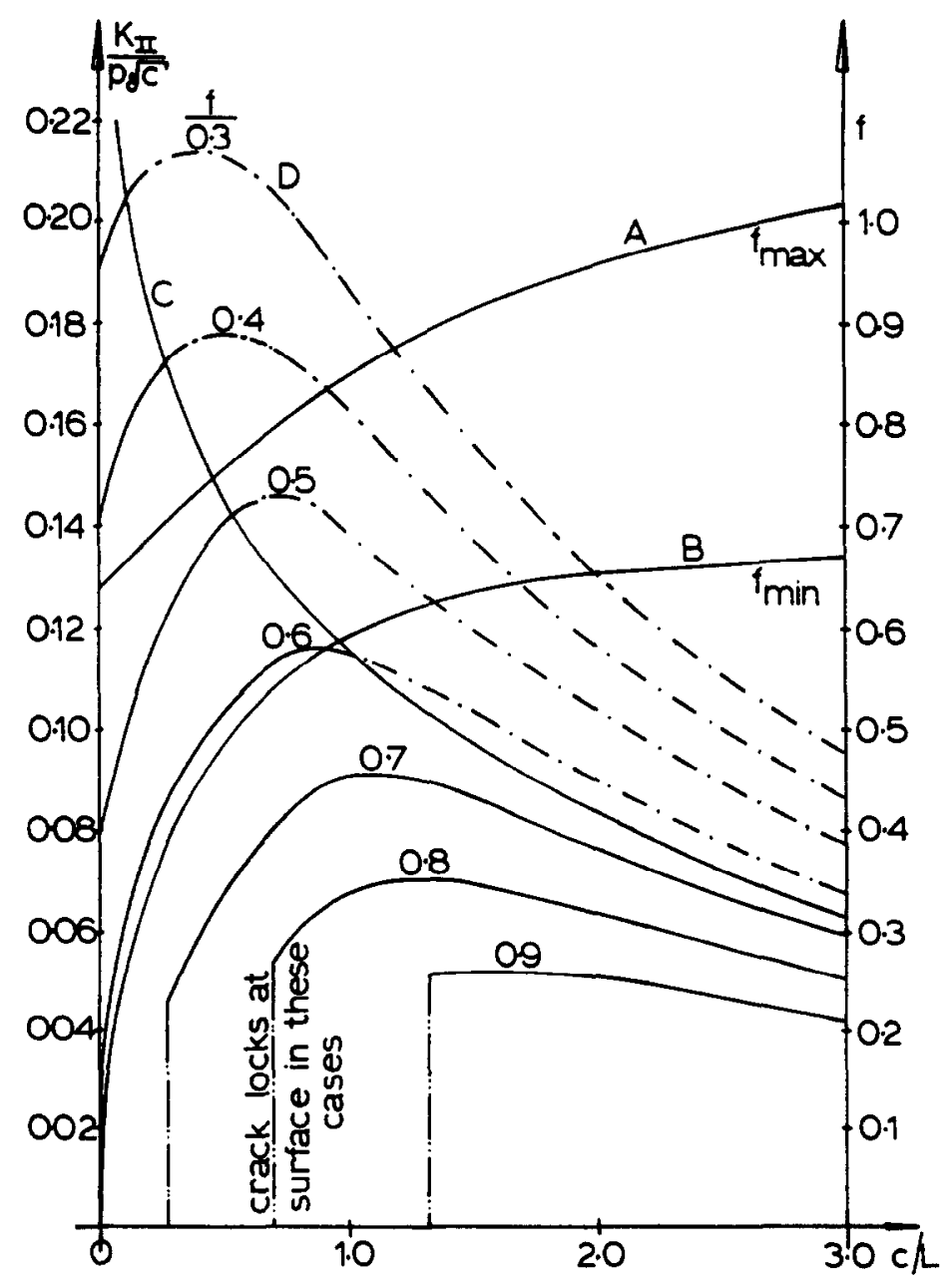

Fig. 2. Crack response regimes and stress intensity factors for $f>f_{\min }$. 
normal tractions ahead of the closure point a. From eqns (2) and (6) we get

$$
\begin{aligned}
& N(x)=p_{0}\left(g_{2}+\lambda g_{3}\right)+\frac{2 \mu}{\pi(\mathrm{k}+1)} \int_{0}^{\prime \prime} B_{y}(\xi) K(x, \xi) \mathrm{d} \xi \\
& N(x)=0, \quad 0<x<a .
\end{aligned}
$$

Since $a<c$, the crack opens smoothly and we require a bounded solution for $B_{y}$. As in [8], we extend the range of definition of $B_{y}$ to an even function along the negative $x$ axis[11], so that we may represent the solution of eqn (11) as

$$
B_{y}(r)=\frac{p_{0}(\kappa+1)}{2 \mu} \psi(r)\left(1-r^{2}\right)^{1 / 2},
$$

where $r=\xi / a$. The resulting equation is discretized by the method of Erdogan et al[ 12]

$$
\begin{aligned}
\sum_{i=1}^{n} \frac{\left(1-r_{i}^{2}\right)}{2(n+1)} \psi\left(r_{i}\right) K\left(s_{k}, r_{i}\right) & =-g_{2}\left(s_{k}\right)-\lambda g_{3}\left(s_{k}\right) \quad k=1,2, \ldots, n+1 \\
r_{i} & =\cos \left[\frac{i \pi}{2(n+1)}\right] \\
s_{h} & =\cos \left[\frac{2 k-1}{4(n+1)} \pi\right] .
\end{aligned}
$$

There are $n+1$ equations for the $n$ unknowns $\psi\left(r_{i}\right)$, and the extra equation, which corresponds to the boundedness condition, is used to determine the value of $a$. The variation of $a / L$ with $\lambda$ is shown in Fig. 3.

The situation regarding the slip zones is more complicated. We distinguish two cases, $f>f_{\min }$ (curve $B$ in Fig. 2) and $f<f_{\min }$, and will discuss them separately.

\section{Case $f>f_{\min }$}

In this case, we find that the forward slip persists to the crack tip after the application of shear. The analysis proceeds as follows: When only the normal load has been applied, the crack faces slip and the tractions transmitted are

$$
\begin{aligned}
N_{o} & =p_{o} g_{2}(x), \quad 0<x<c \\
S(x) & =-f p_{o} g_{2}(x), \quad 0<x<c .
\end{aligned}
$$

The glide dislocation distribution required to accomplish this is the singular solution of the Cauchy integral equation

$$
\frac{2 \mu}{\pi(\kappa+1)} \int_{0}^{c} B_{x}(\xi) K(x, \xi) \mathrm{d} \xi=-p_{o}\left(g_{1}+f g_{2}\right), \quad 0<x<c
$$

The relative tangential displacement between the crack faces is then

$$
h(x)=\int_{x}^{r} B_{x}(\xi) \mathrm{d} \xi
$$

The calculation is described in detail in [8]. We now add the shear load, the crack is allowed to open partially, and the normal traction corrected for the gap, $N^{y}(x)$, is found from eqn (10). Extra glide dislocations are now distributed over the interval $0<x<c$ 
Analysis of fretting fatigue cracks during loading phase

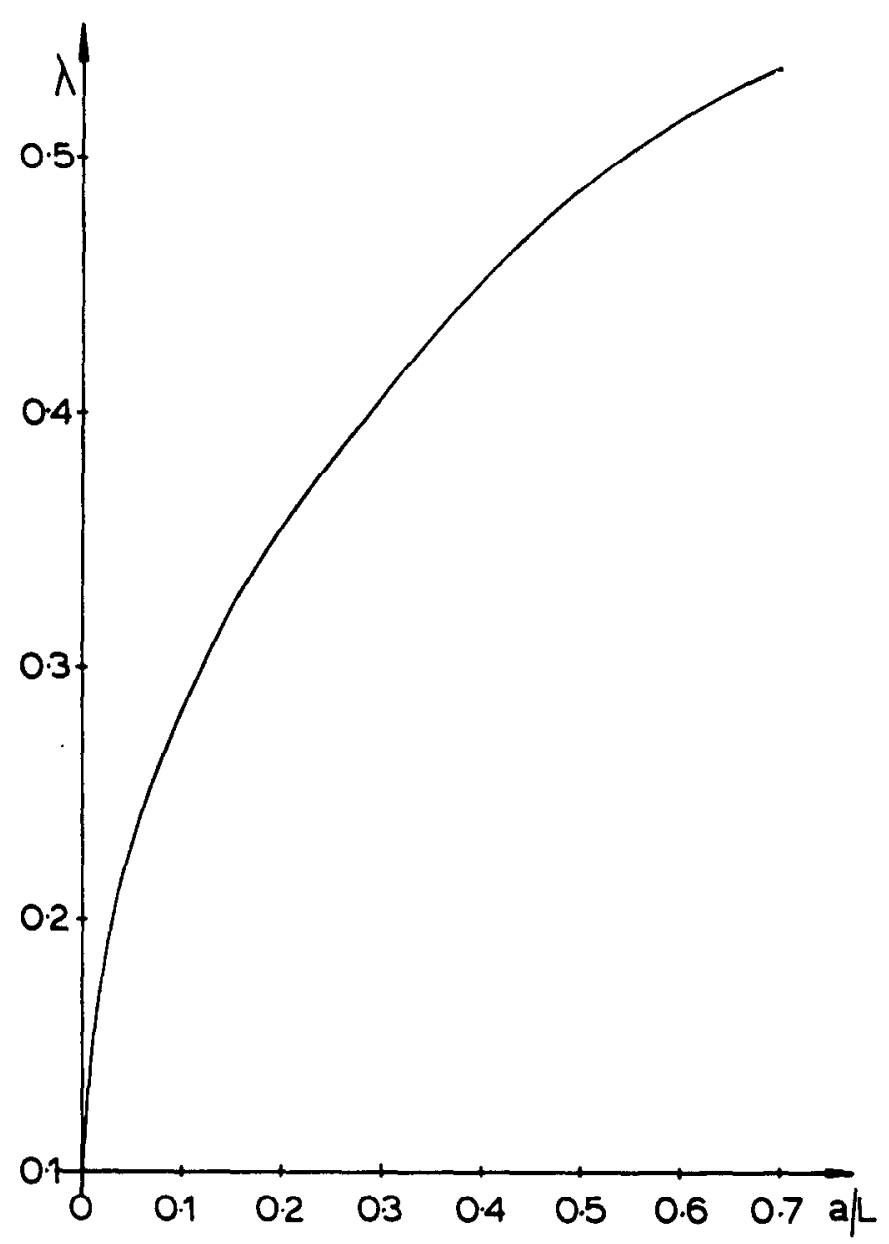

Fig. 3. Variation of crack opening extent with load parameter $\lambda$.

to restore condition (9)

$$
\begin{aligned}
& S(x)=p_{0} g_{2}(\lambda-f)+\frac{2 \mu}{\pi(\kappa+1)} \int_{0}^{c} \hat{B}_{x}(\xi) K(x, \xi) \mathrm{d} \xi \\
& S(x)=-f N^{k}(x), \quad 0<x<c
\end{aligned}
$$

where $\hat{B}_{x}$ is the extra glide dislocation distribution. Equation (21) is treated in the same way as eqn (11), except that we require a singular solution at $x=c$. The discretized form is $[8,11,12]$

$$
\begin{gathered}
\sum_{i=1}^{n} \frac{1}{2 n+1} \hat{\psi}\left(r_{i}\right) K\left(s_{k}, r_{i}\right) \\
=-\frac{1}{c}\left\{f\left[N^{\mu}\left(s_{k}\right) / p_{o}-g_{2}\left(s_{k}\right)\right]+\lambda g_{2}\left(s_{k}\right)\right\} \quad k=1,2, \ldots, n \\
r_{i}=\cos \left[\frac{\pi(2 i-1)}{2(2 n+2)}\right], \quad i=1,2, \ldots, n \\
s_{k}=\cos \left(\frac{\pi k}{2 n+1}\right), \quad k=1,2, \ldots, n .
\end{gathered}
$$

Again the extra relative tangential displacement between the crack faces is found from

$$
\hat{h}(x)=\int_{\cdot}^{\cdot} \hat{B}_{x}(\xi) \mathrm{d} \xi
$$


and for further forward slip to have occurred it is necessary that

$$
\hat{h}(x) \geq 0, \quad 0<x<c
$$

Consequently, the magnitude of the shear stress intensity factor, determined by Krenk's interpolation formula[13], must increase.

\section{Case $f<f_{\min }$}

In this case, the order of events depends on the magnitude of $\lambda$. As the shear load is applied, forward slip extends up to a point $b$ less than $c$, and $b$ is independent of $\lambda$. To see this, imagine that first the normal load is applied and slip prevails up to $c$. When the shear load is applied, the conditions of forward slip and compressive normal traction are violated. Add first a distribution $B_{x}$ to restore the slip condition in the interval $0<x<b$. Then add distributions $B_{y}^{\prime}$ and $-f B_{x}^{\prime}$ in the interval $0<x<a$ to accommodate the gap without violating the slip condition. Using eqns (6) and (7), it is shown that $B_{y}^{\prime}=B_{x}^{\prime}$, and that the equation determining $B_{s}$ is linear in $\lambda$. The boundedness condition then yields $b$ independent of $\lambda$ as in cases of receding contact, which turns out to be the case here. For the numerical computation, we need not follow the steps outlined, but we use eqns (20) and (21) with $c$ replaced by $b$ and require a bounded solution. As the load $\lambda$ increases, the gap increases at the expense of the forward slip zone, since $b$ remains constant. When $a$ reaches $b$, backslip starts. Both cases can be formulated by

$$
\begin{aligned}
& S(x)=p_{\iota,} g_{2}(\lambda-f)+\frac{2 \mu}{\pi(\kappa+1)} \int_{0}^{b} B_{x}(\xi) K(x, \xi) \mathrm{d} \xi \\
& S(x)= \pm f N^{\mu}(x), \quad 0<x<b .
\end{aligned}
$$

In (28) the + sign is used for backslip, and the - sign for forward slip. The symbol $b$ is used to determine the extent of slip in both cases. The numerical procedure is as previously presented and we omit the details.

If $\lambda$ is increased sufficiently, the backslip zone reaches the crack tip $c$. When this happens, the shear stress intensity factor there, introduced at the time of the normal loading and locked-in as $\lambda$ was increased, will now start to diminish as backslip progresses. For this case we must obtain a singular solution to eqn (28) and with $b=c$.

\section{COMPLETELY OPEN CRACK}

If the crack is very short, as $\lambda$ is increased, $a$ will eventually reach $c$ and the gap will be completely open. We then have combined shear and opening mode at the crack tip. We must require that the tractions given by eqns (6) and (7) vanish in the interval $0<x<a$. Singular solutions must be considered. The results are shown in Fig. 6 .

\section{RESULTS}

It is clear by now that the crack response, even during the first quarter of the loading cycle, is very complicated. Depending on the crack length $(c / L)$, the maximum value of $\lambda$, and the value of $f$, various combinations of separation, slip, and backslip may occur as $\lambda$ is increased.

It has been already mentioned that forward slip to the crack tip occurs for $f>$ $f_{\min }$ (curve $B$ in Fig. 2). It transpired from the numerical results that this graph coincides with a graph of $\lambda$ vs. $a / L$ and also with a graph of $f$ vs. $b / L$, where $b$ is the extent of the forward slip. These observations can be proven a priori if we take into account the 


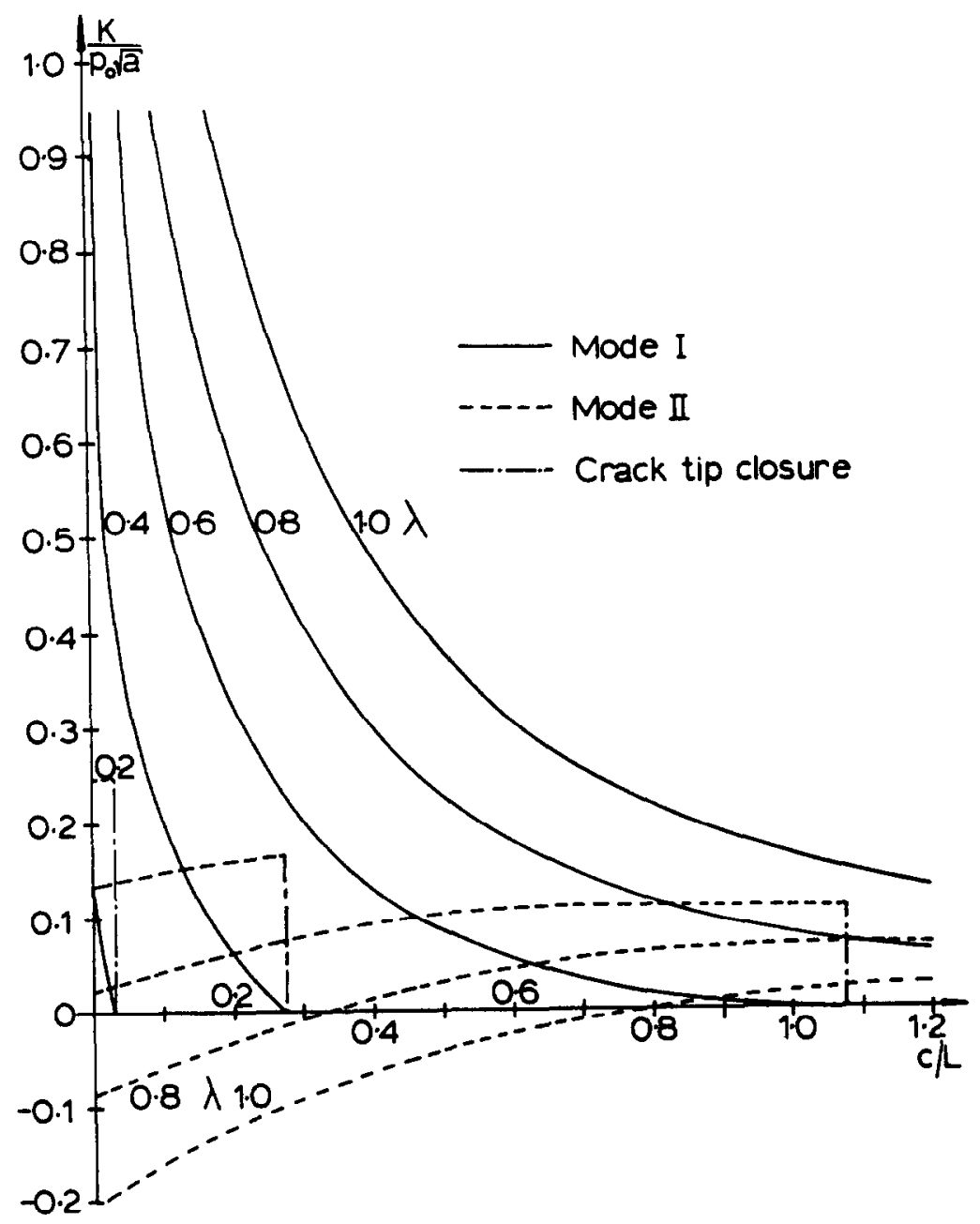

Fig. 6. Stress intensity factors for modes I and II when crack is open to tip.

nature of eqns (6) and (7). The equations that determine $\lambda$ vs. a/L are (10) and (11), with the requirement of a bounded solution. For the forward slip case we follow the same steps as in the argument proving that $b$ is independent of $\lambda$. We find that the glide dislocation distribution required to restore backslip is governed by the same type of integral equation. The right sides of the two equations are proportional if $\lambda$ is identified with $f$. Thus, $\lambda$ vs. $a / L$ is the same as $f$ vs. $b / L$, and in the limit as $b$ approaches $c$ and the solution is still bounded with the crack tip just at the point of sticking, the curve also gives $f_{\min }$ vs. $c / L$. Should $f$ be greater than this value, slip will persist to the crack tip and the stress intensity factor there will be proportional to $\lambda$. Therefore, to reduce the number of graphs necessary to display the results, we record only the value of $K_{\| \prime}$ as $a \rightarrow c$, Fig. 2, curve $C$. For values of $\lambda$ below this limit, $K_{\| \prime}$ may be found by linear interpolation, and for values above, the results are given in Fig. 6 (combined shear and opening mode).

When $f<f_{\min }$, forward slip up to $b$ occurs first. As $\lambda$ increases, the forward slip zone shrinks as $a \rightarrow b$, and in the limit, backslip starts. This happens at $\lambda=f$. The results are shown in Fig. 4. Stress intensity factors are shown for the case of backslip to the crack tip in Fig. 5 . When $a$ reaches $c$, the stress intensity factors become proportional to $\lambda$, as expected.

A limited investigation was carried out for the case of tractions applied simultaneously so that $\lambda$ remains constant. Because of the uncoupled nature of the problem, the quantities associated with the gap are obtained as before. The shear traction dis- 


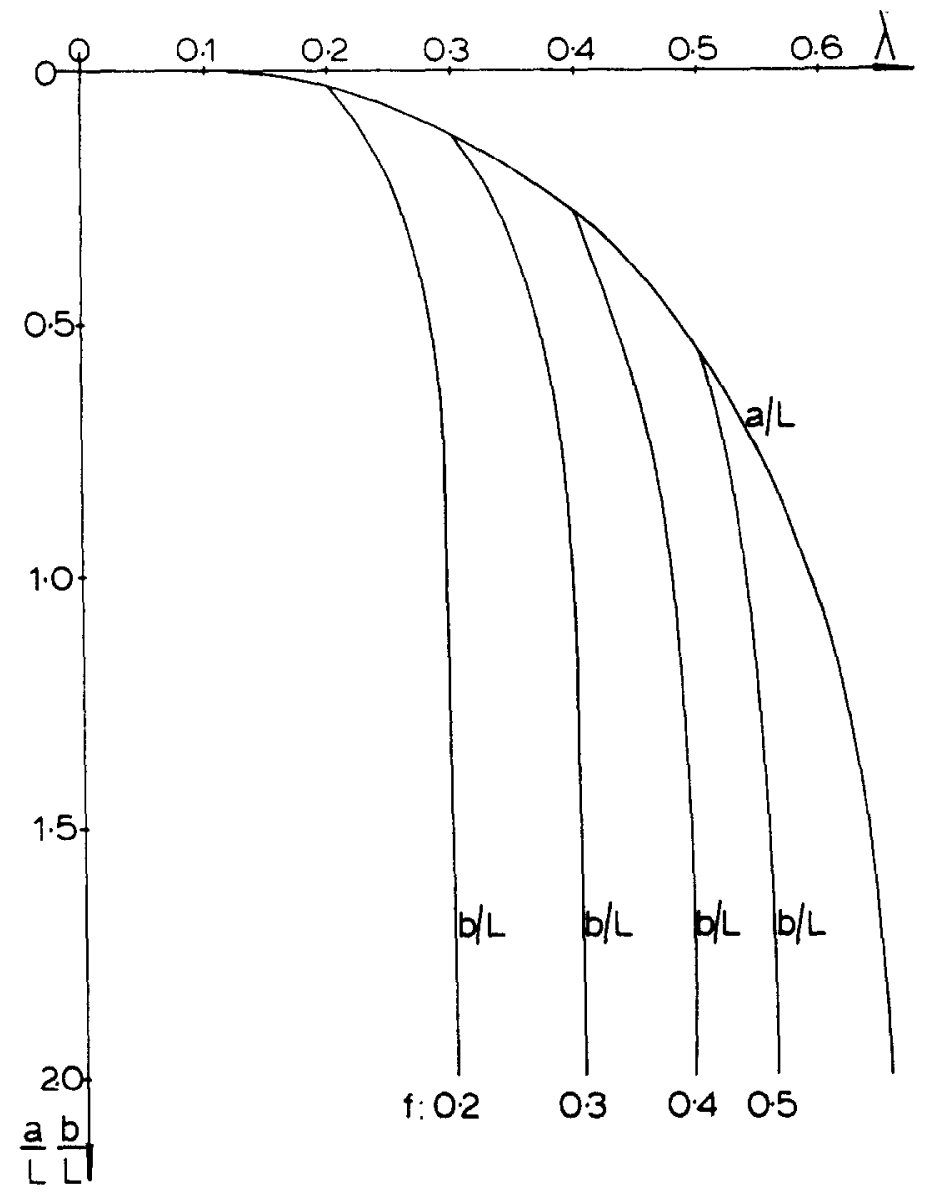

Fig. 4. Depth to which backslip occurs and crack opening extent for several coefficients of interfacial friction.

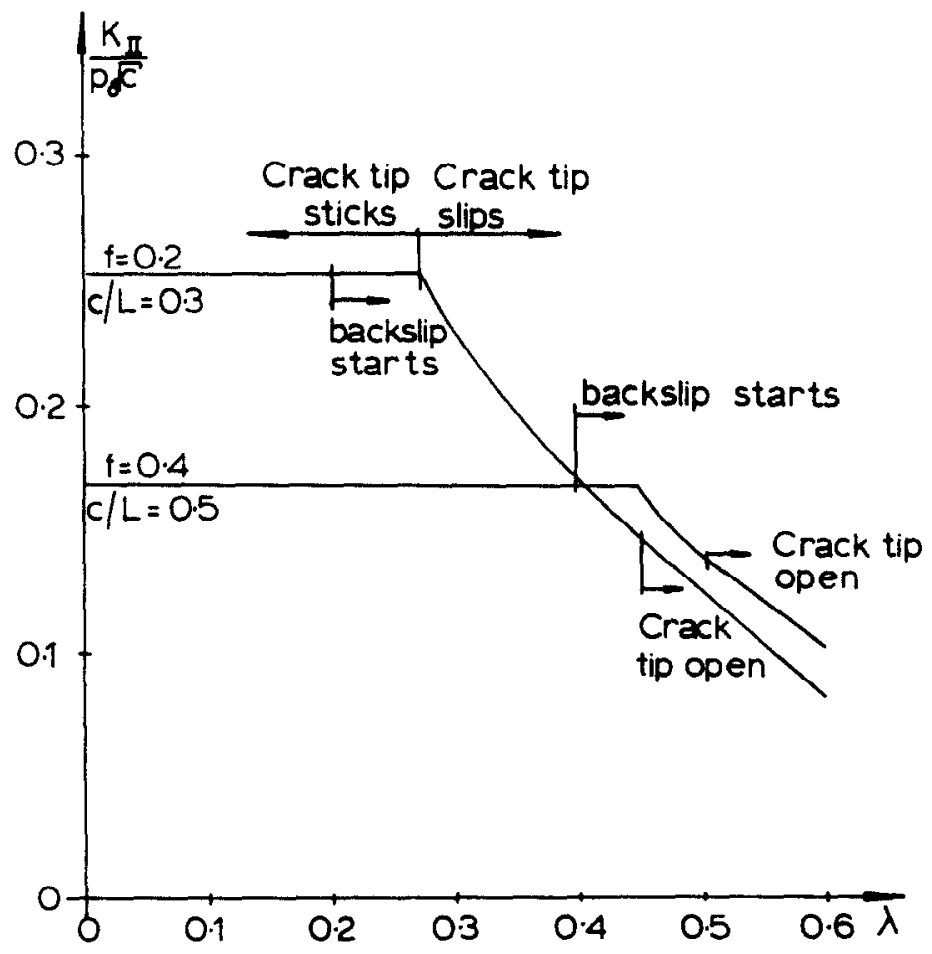

Fig. 5. Crack response in the backslip regime, for two particular cases. showing initial stick. followed by progressive backslip and finally crack-tip opening. 


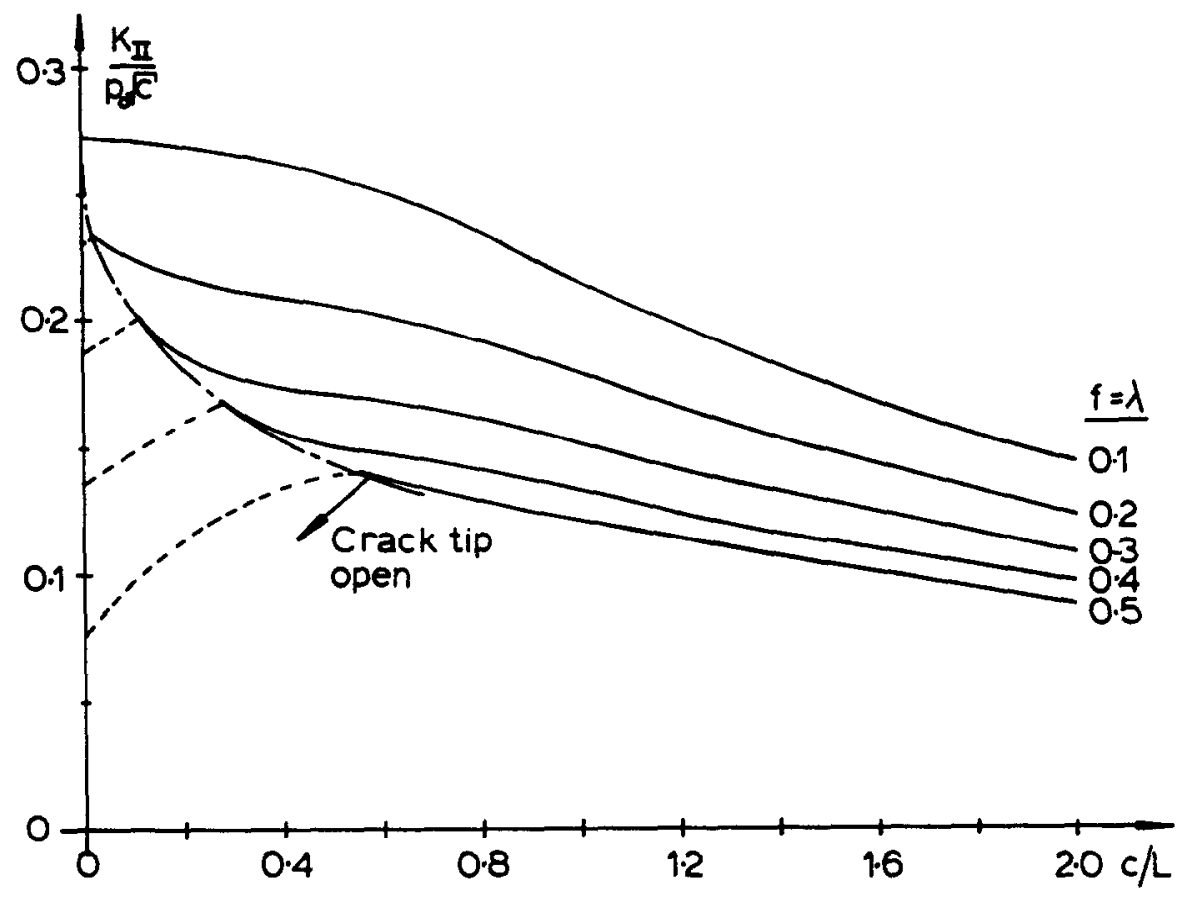

Fig. 7. Stress intensity factors for the special case of $f=\lambda$, when the normal and shear tractions are applied simultaneously and their ratio is held constant.

tribution is obtained from

$$
\begin{aligned}
& S(x)=p_{v}\left(g_{1}+\lambda g_{2}\right)+\frac{2 \mu}{\pi(\kappa+1)} \int_{0}^{c} B_{x}(\xi) K(x, \xi) \mathrm{d} \xi \\
& S(x)=-f N(x) \quad 0<x<c,
\end{aligned}
$$

assuming forward slip to the crack tip. Results are shown in Fig. 7 for the case $f=$ $\lambda$. In eqn (29), $B_{x}$ is the total density.

If only the shear load, $\lambda$, is removed, it is found that forward slip persists, and the values of $K_{1 /}$ increase to those shown by curves $D$, Fig. 2 . It is also verified that the relative tangential slip continues to increase.

\section{CONCLUSION}

Stress intensity factors have been obtained for a wide range of coefficients of friction and crack lengths. The analysis reveals that, for sequential loading, the crack tip experiences a monotonically increasing stress intensity if the interfacial friction is large. If it is small, the crack tip first locks as shear loading is applied, and then experiences a decrease in $K_{11}$, which may fall to zero, and increase in the opposite sense for sufficiently large $\lambda$. In either case, if $\lambda$ is increased to the point where the crack is completely open, the crack tip experiences both shear and opening mode stress intensities.

Acknowledgement-D. Hills would like to acknowledge gratefully the suppon he received by the English Speaking Union during the course of this work.

\section{REFERENCES}

1. R. B. Waterhouse, Frerting Falizue, Applied Sci. Pub. Lld., London (1981).

2. G. P. Wright and J. J. O'Connor, Finite element analysis of alternating axial loading of an elastic plate pressed between two elastic rectangular blocks with friction. Int. J. Engng Sci. 9, 555-570 (1971).

3. G. P. Wright and J. J. O'Connor, The influence of fretting and geometric stress concentrations on the fatigue strength of clamped joints, Proc. Inst. Mech. Engrs. 186, 827-835 (1972). 
4. P. R. Edwards. The application of fracture mechanics to fretting fatigue. In Fretting Fatigue, Applied Science Publishing Lid., London (1981).

5. B. A. Doeser and R. B. Waterhouse. The finite element analysis of a contact undergoing fretting fatigue. Proc. Euromech Coll. No. 110, Contact Problems in Mechanical Assemblages. Linkoping. Sweden (1978).

6. L. M. Keer, M. D. Bryant, and G. K. Haritos, Subsurface cracking and delamination. In Solid Contuct and Lubrication, ASME publication, New York (1980).

7. S. P. Timoshenko and J. N. Goodier. Theory of Elasticity. McGraw-Hill. New York (1970).

8. D. A. Hills and M. Comninou, A normally loaded half-plane with an edge crack. Int. J. Solids Structures, in press.

9. J. Dundurs and G. P. Sendeckyj, Behavior of an edge dislocation near a bimetallic interface. J. Appl. Phys. 36, 3353-3354 (1965).

10. J. Dundurs and T. Mura, Interaction between an edge dislocation and a circular inclusion. J. Mech. Phys. Solids 12, 177-189 (1964).

11. G. D. Gupta and F. Erdogan, The problem of edge cracks in an infinite strip. J. Appl. Mech. 41, 10011006 (1974).

12. F. Erdogan, G. D. Gupta, and T. S. Cook, Numerical solution of singular integral equations. In Methods of Analysis and Solutions of Crack Problems (Edited by G. C. Sih), Noordhoff, Leyden (1973).

13. S. Krenk, On the use of the interpolation polynomial for solutions of singular integral equations. Q. Appl. Maths. 32, 479-484 (1975). 